\title{
ANALYSIS OF THE FOUNDATION SLAB SETTLEMENT OF THE “AFRICA PAVILION” FACILITY IN WROCŁAW
}

\author{
EUGENIUSZ SAWICKI, TOMASZ STRZELECKI
}

Faculty of Civil Engineering, Wrocław University of Technology,

Wybrzeże Wyspiańskiego 27, 50-370 Wrocław, Poland

\begin{abstract}
This article presents the results of numerical calculations of soil consolidation underneath the "Africa Pavilion" structure in Wrocław Zoo, Poland. To determine the deformations of the baseplate of the "Africa Pavilion" and deformations of the subsoil, Biot's consolidation theory for two-phase medium was applied. The calculations were carried out using the professional program FlexPDE v.6, which is based on the Finite Element Method. Numerical calculations performed were used to evaluate the design assumptions allowing for the laying of hydraulic conduits under the slab.
\end{abstract}

Key words: soil consolidation, numerical calculations, settlement, hydraulic conduits

\section{INTRODUCTION}

The issue of settlement of foundations is one of the main elements of serviceability of the structure analysis. The size of settlements, their uniformity and variability in time have a significant impact on the conditions that determine the functional requirements of the structure. For this reason, each foundation design should include an analysis of foundation settlement. In geotechnics practice, the most commonly used models to perform such analysis are Terzaghie's consolidation model or Winkler's model. However, in the case of more complex issues, such an approach may not be sufficient. For this reason, Biot's consilidation model [16] is of great interest and it is still being studied and developed, e.g., [1], [2], [11], [12]. Poro-elastic theory may be applied in a great variety of fields, ranging from reservoir engineering to biomechanics [9]. For example, this serves in environmental issues such as groundwater withdrawal and land subsidence, e.g., [15]. In biomechanics, Biot's theory is used to describe the bone deformation under a mechanical load [14]. Nevertheless, the numerical solution to the Biot

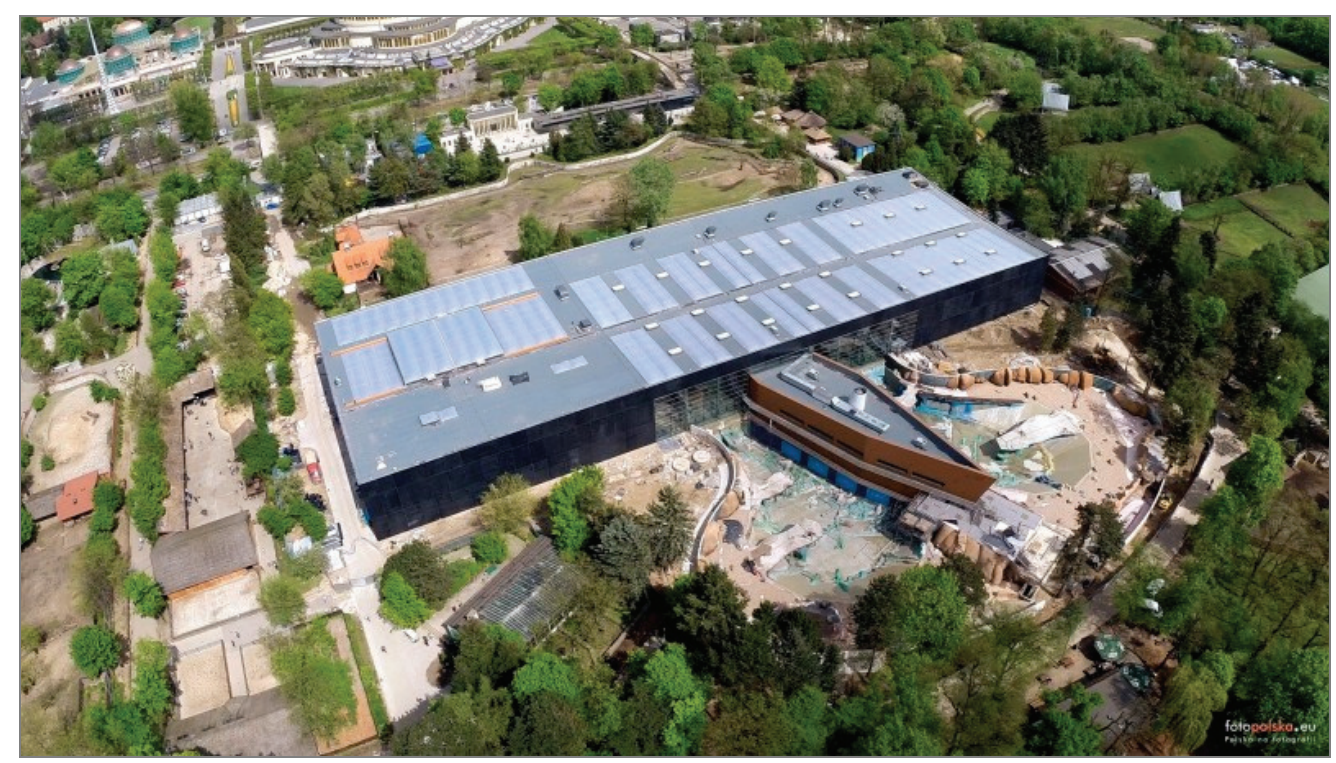

Fig. 1. “Africa Pavilion” complex in Wrocław Zoo (bird's-eye view; photo from [17]) 


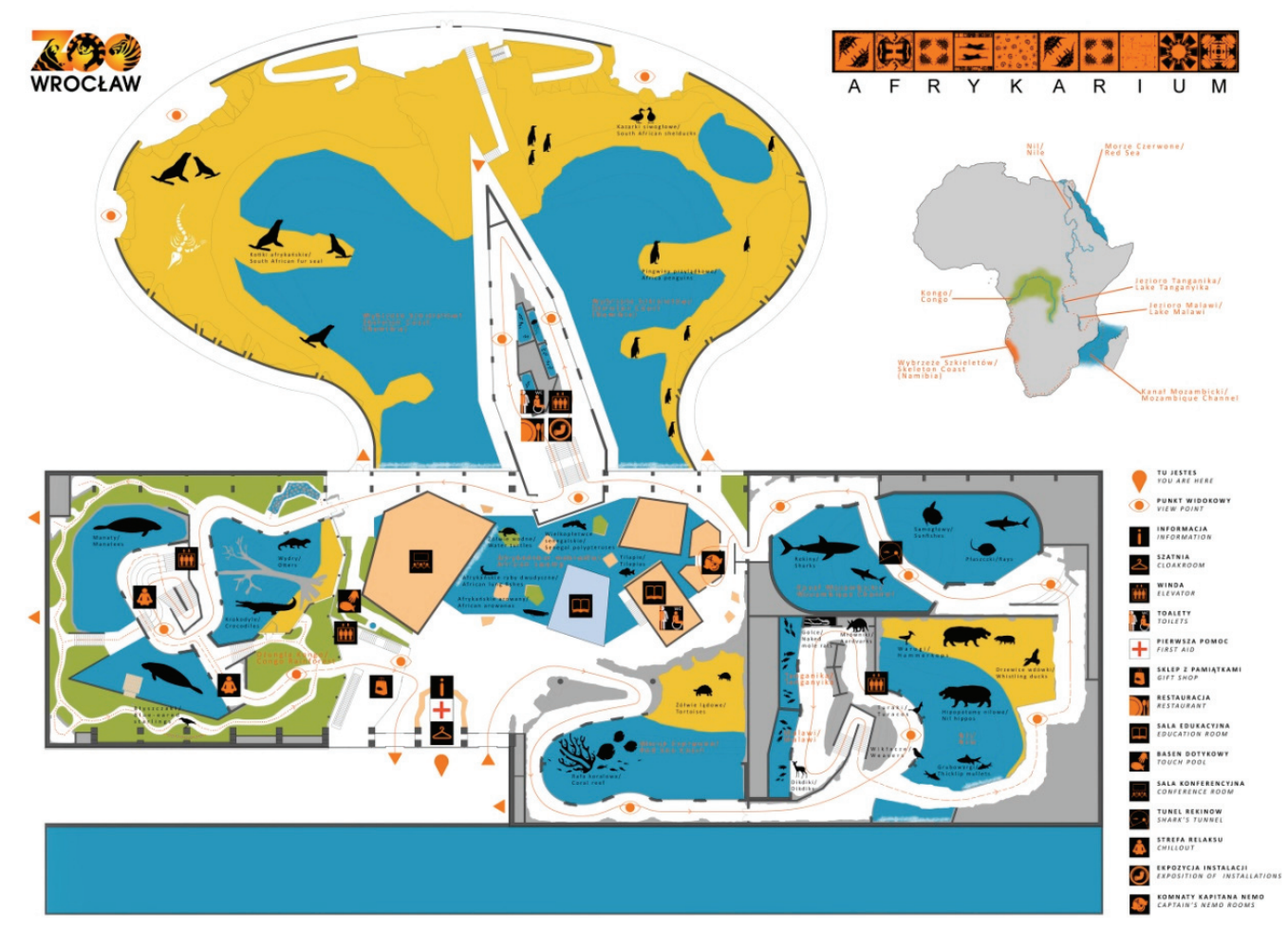

Fig. 2. Animals of the "Africa Pavilion" complex (photo from [17])

partial differential equations is difficult and still remains a challenge [9].

In this paper, the authors present the settlement analysis of the baseplate of the "Africa Pavilion" facility in Wrocław, based on Biot's consolidation theory for a two-phase medium [3], [4]. The "Africa Pavilion" is the complex (Fig. 1 and Fig. 2) containing various environmental ecosystems of Africa. There are 19 swimming pools and reservoirs in total including, among others: coral reefs of the Red Sea, Nile hippos, freshwater fish from Malawi and Tanganyika Lakes, fauna from the jungle surrounding the Congo River like crocodiles and manatees.

For these exotic fauna and flora to exist, it is necessary to supply water and nutrients to the individual ecosystems. This task is achieved by the so-called "lifeline system", i.e., a system of pipes distributed under the foundation slab. There is, therefore, a danger of damage to the system caused by settling "Africa Pavilion" building, a detailed analysis of settlement of the building was thus deemed necessary.

To fulfill the aim, a spatial numerical model of Biot consolidation for two-phase medium was built. This model allows the simulation of mechanical processes in the ground and in the baseplate. An important element of the model is the assumption that both the soil and reinforced concrete are porous media through which water or gas can percolate. The adopted model is linear, both in terms of the theory of elasticity and filtration of water in the porous medium.

\subsection{GEOLOGICAL AND HYDROGEOLOGICAL CONDITIONS}

Eleven boreholes and fourteen cone penetration tests were made in the area of the future construction site. Boreholes and test holes were max. 15 meters deep.

The first layer of the soil consists of heterogeneous material. Its thickness is from 1 to $2.6 \mathrm{~m}$. Deeper down, there are clay and clay with sand, their thicknesses varying between 0.3 and $2.4 \mathrm{~m}$. Below this level one finds clay and in many places, directly underneath heterogeneous material like sand, sand with gravel and gravel with cobble. The bottom of these coarse-grained soils goes down to $10.6-12.0 \mathrm{~m}$ below the surface. Clay also occurs in the coarse-grained soils, the thickness of the inclusions ranges between 0.4 and $2.2 \mathrm{~m}$. A further layer of glacial clay is found underneath the coarse-grained soils. The bottom of the last layer was not drilled (the bottom is deeper than 15 meters).

The aquiferous layers are formed from: medium sand, sand with gravel, gravel, and gravel with cobble. As described above, the aquiferous layers are 
both confined and unconfined. The depth of the water table ranges between ordinates 115.2 and 115.4 above sea level (about 2.6 to 2.8 below the ground surface).

\subsection{PREPARATORY WORK TO DETERMINE THE DEFORMATIONS OF THE SLAB AND METHODOLOGY OF CALCULATIONS AND RESULTS}

One of the most important issues when calculating deformations of the slab and settlement of the soil around the slab is the choice of an appropriate mathematical description of these processes. In this case, the spatial numerical model of consolidation of a poroelastic medium (Biot's medium) was created. This model allows the simulation of mechanical processes in the slab and in the soil around the slab. The adopted model is linear both in the range of theory of elasticity and in the range of water flow through the porous matrix. The important assumption that water can percolate, not only through soil but also through the slab, was also made.

In order to build the model the following tasks were performed:

(a) studies of geology and hydrogeology of the construction site, construction of the slab, loads of the slab, parameters of geotechnical layers of the soils, and already registered slab deformations were performed,

(b) a digital terrain model (DTM), containing the slab and construction site together with surrounding area, was created,

(c) the mathematical description of the consolidation (Biot model) of soil and deformation of the slab was adopted,

(d) the boundary and initial conditions of the problem were formulated,

(e) the appropriate script (a mathematical description of the problem in a form accepted by software) for FlexPDE was implemented,

(f) numerical calculations for different combinations of boundary conditions were performed; results of displacement of soil and the slab, as well as results of stress state in soil and in the slab, were registered,

(g) visualisation of displacements of the slab by introducing the results of numerical calculations to DTM was made,

(h) calibration of the numerical model by modification of mechanical parameters and permeability coefficients for geotechnical layers was performed.

\section{MATHEMATICAL MODEL OF BIOT-DARCY CONSOLIDATION}

As previously mentioned, the consolidation model of Biot-Darcy was a mathematical model adopted to determine the deformations of the slab and the settlement of the soil. To use this model the following basic assumptions ([3], [4], [13]) were made:

- soil is a two-phase medium; this medium consists of the elastic soil matrix and the compressible viscous liquid in the pores,

- porous medium is homogeneous and isotropic,

- deformations of soil matrix are small, hence the elements of strain tensor are in the form:

$$
\varepsilon_{i j}=\frac{1}{2}\left(\frac{\partial u_{i}}{\partial x_{j}}+\frac{\partial u_{j}}{\partial x_{i}}\right),
$$

- stress $\sigma_{i j}$ in the soil matrix is not related to grain surface of soil but to the whole surface $d S$ of porous medium. The relation $\sigma=-f p$ is valid for liquids; where the hydrostatic pressure of the liquid in the pores of soil is denoted by $p, f$ is the volumetric porosity of the medium, and $\sigma$ represents the total normal tension applied to the fluid part of the surface $d S$,

- the volumetric porosity of the medium $f$ is a statistical parameter which is supposed to be constant,

- to process this description, the Eulerian frame of reference was adopted.

Constitutive equations for consolidation model are in the form originally proposed by Biot ([4], [5])

$$
\begin{gathered}
\sigma_{i j}=2 N \varepsilon_{i j}+(A \varepsilon+Q \theta) \delta_{i j}, \\
\sigma=Q \varepsilon+R \theta .
\end{gathered}
$$

The constants presented in the above equations have the following meaning:

- $N$ is the shear modulus of the matrix,

- $A$ is the bulk modulus of the matrix when its spaces are filled by liquid,

- $Q$ is the coefficient representing the coupling between the volume change of the matrix and the pressure in the liquid,

- $R$ is the bulk modulus of the liquid which fills the pores of the medium (matrix spaces),

- $M$ is defined as: $M=A-\frac{Q^{2}}{R}$.

The constants $M$ and $N$ correspond to the Lame coefficients in the theory of elasticity: $\lambda$ and $\mu$, 
respectively [7]. On the basis of [6], [13] the constants $M$ and $N$ can be expressed as a function of the shear modulus $G$ and Poisson's coefficient $v$

$$
\begin{gathered}
N=G, \\
A=\frac{2 v G}{(1-2 v)} .
\end{gathered}
$$

Finally the Biot-Darcy set of equations of the linear theory of consolidation where the process can be considered as quasi-static is presented as follows

$$
\begin{gathered}
N \nabla^{2} u_{i}+(M+N) \varepsilon_{, i}+X_{i} \rho=-\frac{H}{R} \sigma_{, i}, \\
C \nabla^{2} \sigma+X_{i} \bar{\rho}=\frac{1}{R} \dot{\sigma}-\frac{H}{R} \dot{\varepsilon},
\end{gathered}
$$

where

$$
H=Q+R, \quad C=\frac{k}{f^{2} \rho_{l} g}, \quad M=A-\frac{Q^{2}}{R},
$$

and $k$ is Darcy's coefficient.

\section{SIMPLIFYING ASSUMPTIONS AND EFFECTIVE PARAMETERS}

Due to the short construction time of the "Africa Pavilion" complex, the primary consolidation of the system slab-soil was taken into account. The values of mechanical parameters for geological layers and for the slab, to predict the settlements, were adopted as shown in Table 1. Biot's constants $(N, A, Q, R)$ were adopted from literature, for example, [8]. Friction angle, cohesion and Darcy's coefficient for geological layers were developed from valid norms and literature, based on simple indexes from in-situ tests. Specific weight was determined from laboratory tests.
Values of Darcy's coefficient for every layer are presented in Table 2 .

Table 2. Darcy's coefficients for layers and for the slab

\begin{tabular}{|l|c|}
\hline \multicolumn{1}{|c|}{ Layer } & $\begin{array}{c}\text { Darcy's } \\
\text { coefficient } \\
k \\
{[\mathrm{~m} / \mathrm{s}]}\end{array}$ \\
\hline Clay & $5 \mathrm{e}-9$ \\
\hline Sand with gravel & $5 \mathrm{e}-4$ \\
\hline $\begin{array}{l}\text { Reinforced } \\
\text { concrete slab }\end{array}$ & $1 \mathrm{e}-23$ \\
\hline
\end{tabular}

The different levels of slab foundation and distribution of load were mapped in the calculation process.

\section{DISCRETIZATION AREA, BOUNDARY CONDITIONS AND RESULTS OF NUMERICAL SIMULATIONS}

The numerical model used in simulations was built as 3D model. It takes into account the layer of sand with gravel and layer of clay. The different levels of the slab foundation were also included.

The system's foundation slab and soil, implemented in Flex PDE ([10]), generated a finite elements mesh consisting of 408,986 nodes, which created 284,347 trihedral elements in space. It corresponds to $1,635,944$ unknowns:

- three components of the displacement vector, - stress of water $\sigma$ in the pores of the soil medium.

The image of the slab directly on the construction site and spatial image of numerical model are presented in Fig. 4a and 4b, respectively.

Table 1. Initial values of parameters for geological layers and for the slab

\begin{tabular}{|l|c|c|c|c|c|c|c|}
\hline \multicolumn{1}{|c|}{ Layer } & $\begin{array}{c}N \\
{[\mathrm{~Pa}]}\end{array}$ & $\begin{array}{c}A \\
{[\mathrm{~Pa}]}\end{array}$ & $\begin{array}{c}Q \\
{[\mathrm{~Pa}]}\end{array}$ & $\begin{array}{c}R \\
{[\mathrm{~Pa}]}\end{array}$ & $\begin{array}{c}\text { Friction } \\
\text { angle } \\
{\left[{ }^{\circ}\right]}\end{array}$ & $\begin{array}{c}\text { Cohesion } \\
{[\mathrm{Pa}]}\end{array}$ & $\begin{array}{c}\text { Specific } \\
\text { weight } \\
{\left[\mathrm{kg} / \mathrm{m}^{3}\right]}\end{array}$ \\
\hline Multiplier & $1 \mathrm{E}+06$ & $1 \mathrm{E}+06$ & $1 \mathrm{E}+06$ & $1 \mathrm{E}+09$ & 1 & $1 \mathrm{E}+03$ & 1 \\
\hline Clay & 26.92 & 40.38 & 58.33 & 2.21 & 21 & 40 & 2100 \\
\hline Sand with gravel & 80.00 & 120.00 & 173.33 & 2.21 & 41 & 0 & 2100 \\
\hline $\begin{array}{l}\text { Reinforced } \\
\text { concrete slab }\end{array}$ & 46153.85 & 69230.77 & 10000.00 & 2.21 & & & 2600 \\
\hline
\end{tabular}




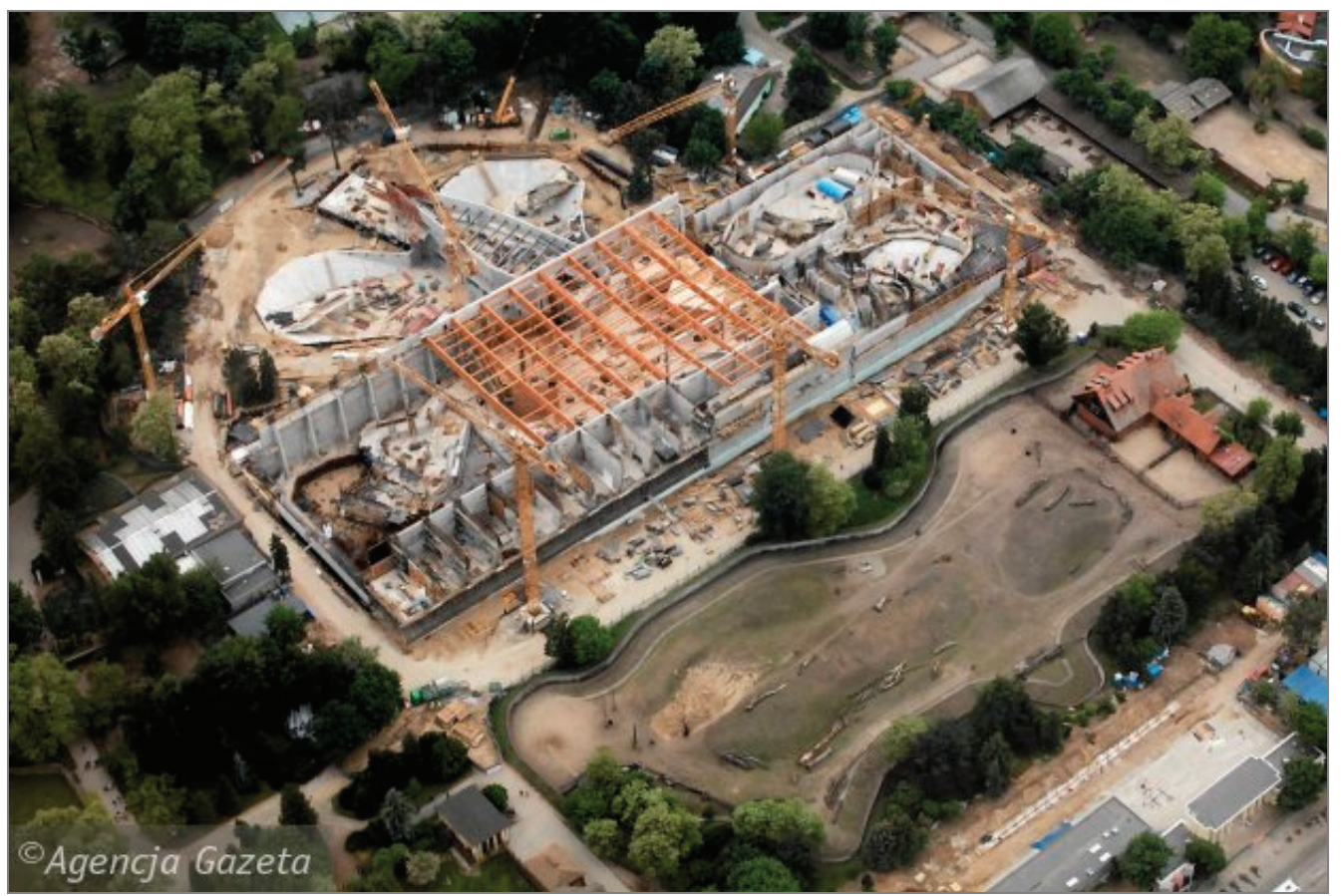

Fig. 4a. Foundation slab of the "Africa Pavilion" complex (photo from [17])

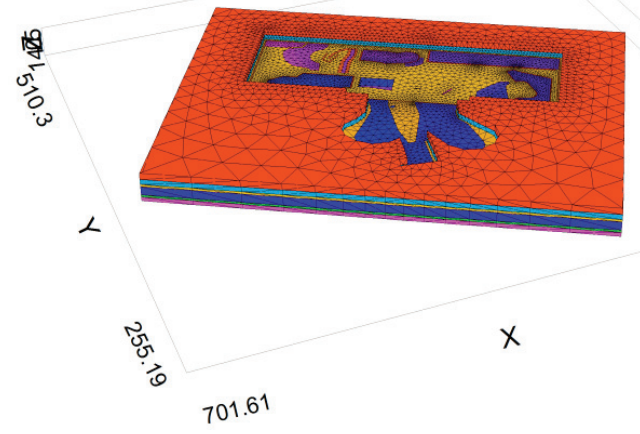

Fig. 4b. Spatial image of the numerical model used to predic settlement of soils and foundation slab deformations

The mathematical model allows the calculation of stress and strain state in the ground and in the slab. It also defines the vectorial field of displacement of the slab and of the surrounding soil. The slab was loaded with concentrated forces (pillars) and by distributed forces (walls, soil load, water load). However, forces originating from pillars were assembled in a few groups and imposed as distributed forces of different values. The schema of slab load is presented in Fig. 5. The different colours represent different types and values of load. There are also the cross-sections A1-A1' (west-east) and B1'-B1 (north-south) marked, for which the edition of calculations results were made. The results from Flex PDE presented in the figures are expressed in the basic SI units (m, Pa).

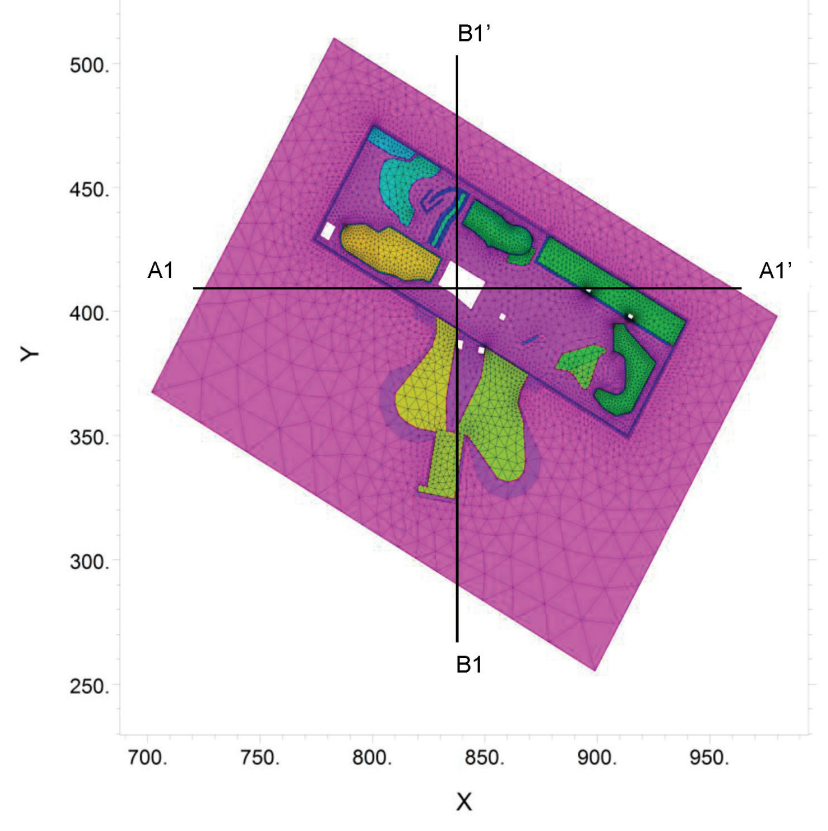

Fig. 5. Mesh of finite elements at the level of the slab. Scheme of slab load; different colours represent different types and values of load.

White areas represent the lower level of the slab

For the purpose of computing, the area of interest was divided into six layers of the seven planes (see Fig. 6 and Fig. 7):

1. The terrain surface ordinate is $118.7 \mathrm{~m}$ above sea level (a.s.1.).

2. The groundwater table is $115.2 \mathrm{~m}$ a.s.1. 
3. The top surface of the foundation slab lies at $112.7 \mathrm{~m}$ a.s.1.

4. The bottom surface of the foundation slab lies at $111.5 \mathrm{~m}$ a.s.1.

5. The top surface of the slab pockets is $107.2 \mathrm{~m}$ a.s.l.

6. The bottom surface of the slab pockets, and the boundary between clay and sand with gravel, is at the ordinate $106 \mathrm{~m}$ a.s.l.

7. The reference level is $104 \mathrm{~m}$ a.s.l.

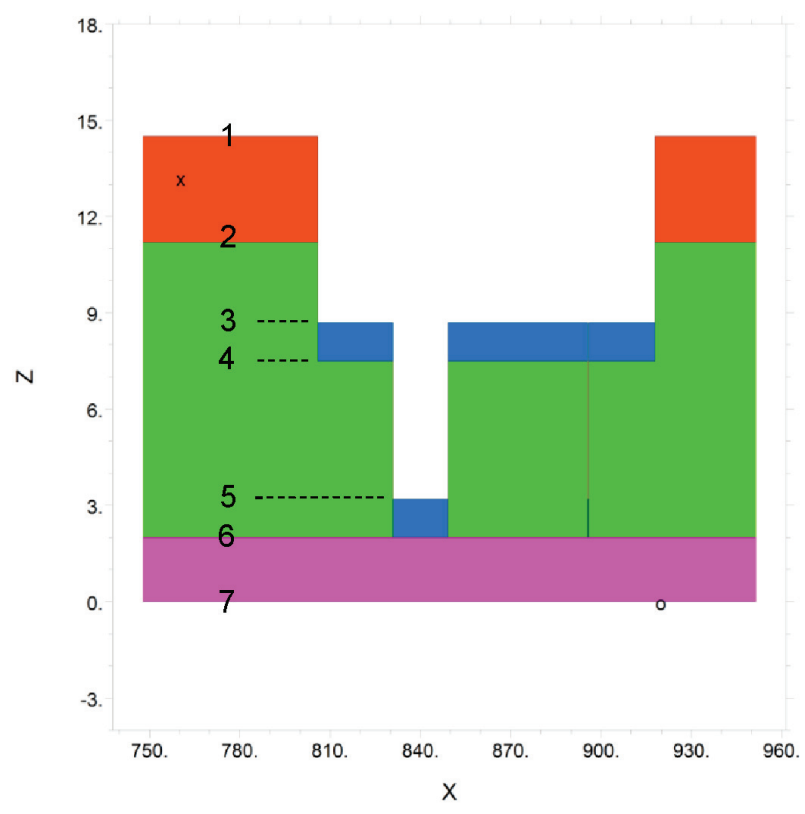

Fig. 6. Distribution of computing layers in the cross-section west-east A1-A1'. (More detailed description is in the text)

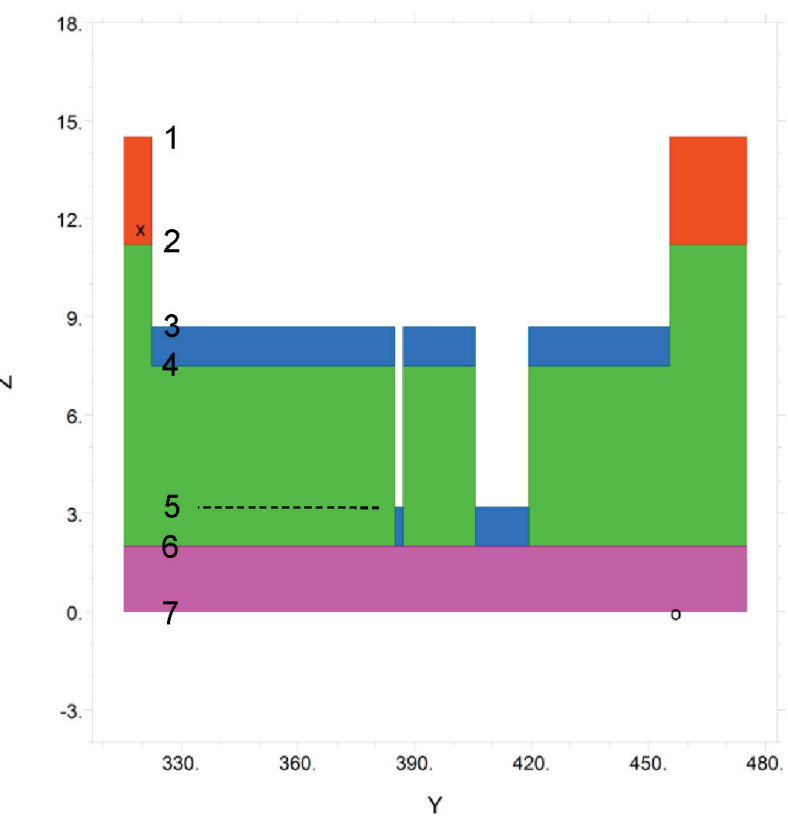

Fig. 7. Distribution of computing layers in the cross-section south-north B1-B1'. (More detailed description is in the text)
The cross-sections through computing layers from west to east A1-A1' and from south to north B1-B1' are shown in Fig. 6 and 7, respectively. Colours assigned to individual layers have the following meaning:

- red defines the sand with gravel above of the groundwater surface,

- green defines the sand with gravel in the saturation zone,

- pink defines the layer of clay,

- blue defines the foundation slab.

Moreover, in the whole domain, the following initial boundary conditions were adopted:

- the displacements: $u, v, w$ of porous medium along the axes: $x, y, z$, respectively, were equal to zero,

- the water pressure in the pores $\sigma$ was equal to the hydrostatic pressure.

Additionally at the reference level (surface 7; Figs. 6 and 7) the displacements: $u, v, w$ were fixed and equal to zero. Similarly at the external "side wall" of the domain the displacements along the axes: $x, y$ were fixed and equal to zero, too.

\section{Stress state in the soil and in the slab}

Calculation results provide the full stress tensor. However, the main diagonal of stress tensor and variation of its components in the soil and in the slab are important in this analysis.

Stress ranges in both cross-sections are of the same order of magnitude. As we can see from the charts, significant changes occur in the slab.

Now, when the normal components of the stress tensor are known, it is possible to verify soil stability. To this purpose, the plastic potential was calculated using Drucker-Prager criteria. The outcomes show that in the soil, in the whole domain of interest, the plastic potential does not change the sign. This means that soil remains in the range of elastic behaviour. It can, therefore, be considered that the adoption of the elastic model of soil (Biot's model) is correct and that a term representing plastic behavior of soil is not required. In Fig. 8, the plot of plastic potential in the cross-section $\mathrm{B} 1-\mathrm{B} 1$ ' is presented.

To calculate the values of plastic potential, according to the Drucker-Prager criterion, the following formula was used

$$
G=A \cdot J_{1}+\sqrt{J_{2}}-k h s,
$$

where

$$
\begin{aligned}
& A=3^{*} \operatorname{sqrt} /(0.33) /(\sin \varphi), \\
& k h s=2^{*} c /(\sin \varphi), \\
& c \text { is the cohesion of soil, } \\
& \varphi \text { is the angle of internal friction. }
\end{aligned}
$$




$$
\begin{gathered}
J_{1}=\sigma_{x x}+\sigma_{y y}+\sigma_{z z}, \\
J_{2}=\sqrt{\frac{\left(\sigma_{x x}-\sigma_{y y}\right)^{2}+\left(\sigma_{x x}-\sigma_{z z}\right)^{2}+\left(\sigma_{z z}-\sigma_{z z}\right)^{2}}{6}}+\sigma_{x y}^{2}+\sigma_{x z}^{2}+\sigma_{y z}^{2} .
\end{gathered}
$$

As we can observe in Fig. 8, the plastic potential in the existing state of stress does not change the sign. Hence, we can conclude that the process of plastic deformation of the ground and baseplate is not present in the study area.

Zero value for the whole soil in Fig. 8 is due to scale selection.

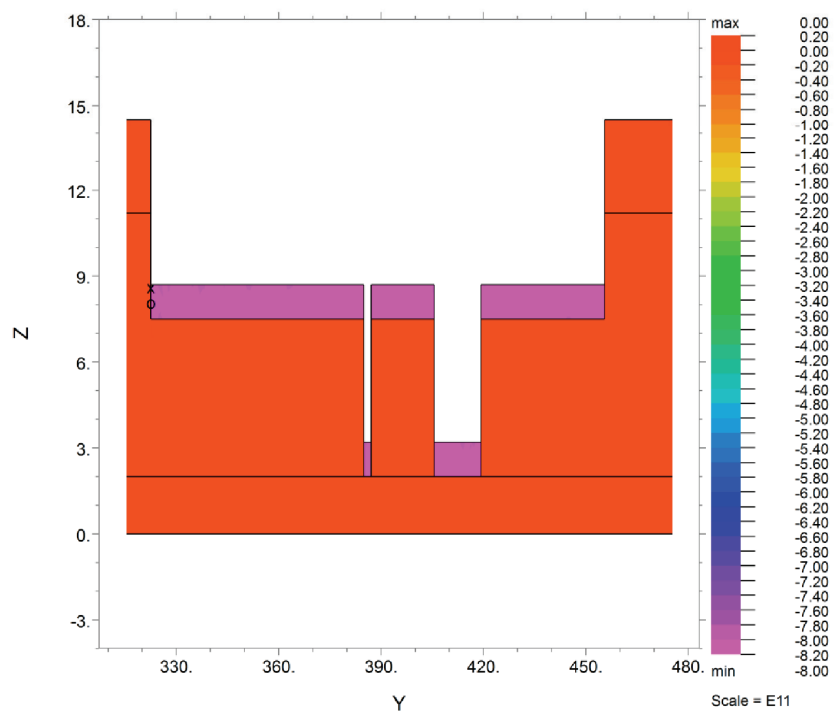

Fig. 8. Plastic potential in the cross-section B1-B1' according to the Drucker-Prager criterion

\section{Settlement of the slab with soil and vectorial field of displacements}

In Fig. 9, the isolines of vertical displacements of the top surface of the area are presented.

The maximum value of settlement is equal to $1 \mathrm{~cm}$. As we can observe, there is a large variability of settlements depending on location point.

The spatial image of settlements of the foundation slab together with soil shown in Fig. 10 looks quite interesting. In this case, the vertical dimension (settlements) is about 1000 times greater than horizontal dimension (ratio 1000:1).

In Fig. 11, the vectorial field of horizontal (in plane $x, y$ ) displacements at the top of foundation slab is presented. The values of horizontal displacements are very small. The maximum value is equal to $0.626 \mathrm{~mm}$.

The vectorial field of vertical displacements for cross-sections A1-A1' and B1-B1' is shown in Figs. 12 and 13 , respectively.

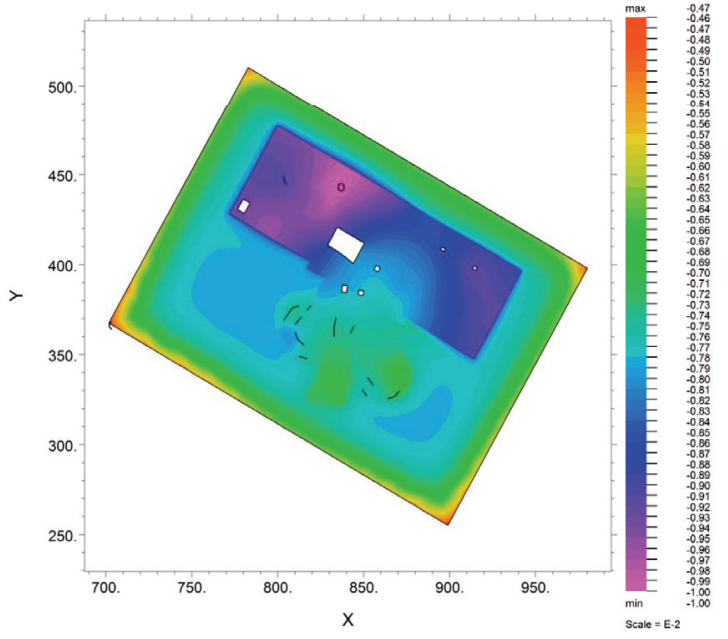

Fig. 9. Isolines of settlement of the top surface of the foundation slab. (Settlement is expressed in meters; scale multiplier $=1 \mathrm{e}-2$ )

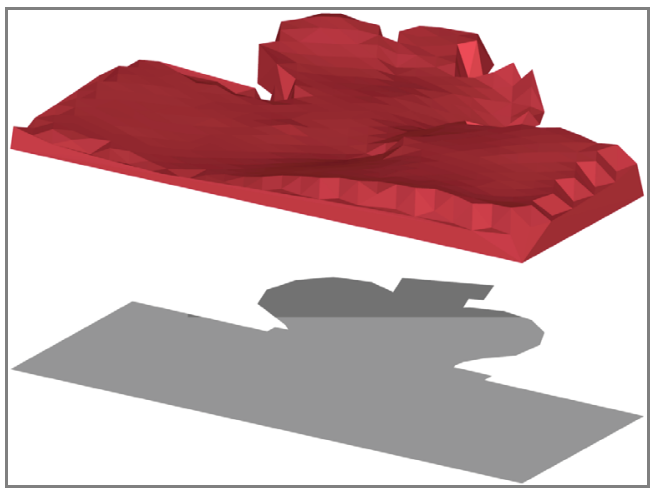

Fig. 10. A spatial image of settlements of the foundation slab

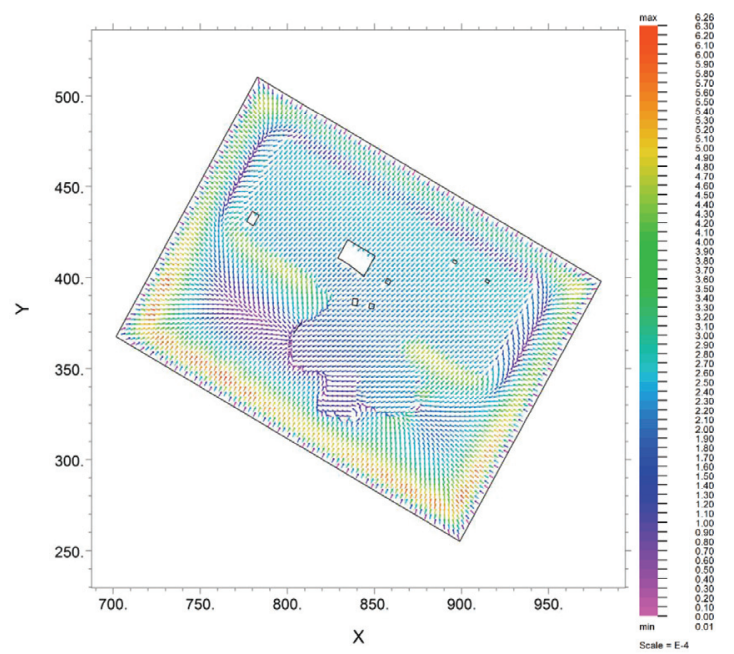

Fig. 11. The vectorial field of horizontal displacements at the top of the foundation slab.

$($ Displacements are expressed in meters; scale multiplier $=1 \mathrm{e}-4)$ 


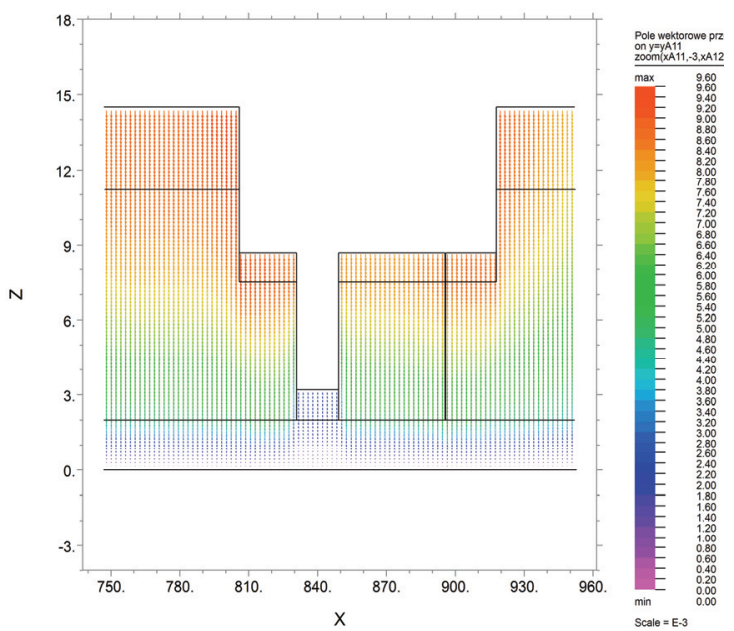

Fig. 12. Vectorial field of vertical displacements in the cross-section A1-A $1^{\prime}$

$($ Displacements are expressed in meters; scale multiplier $=1 \mathrm{e}-3)$

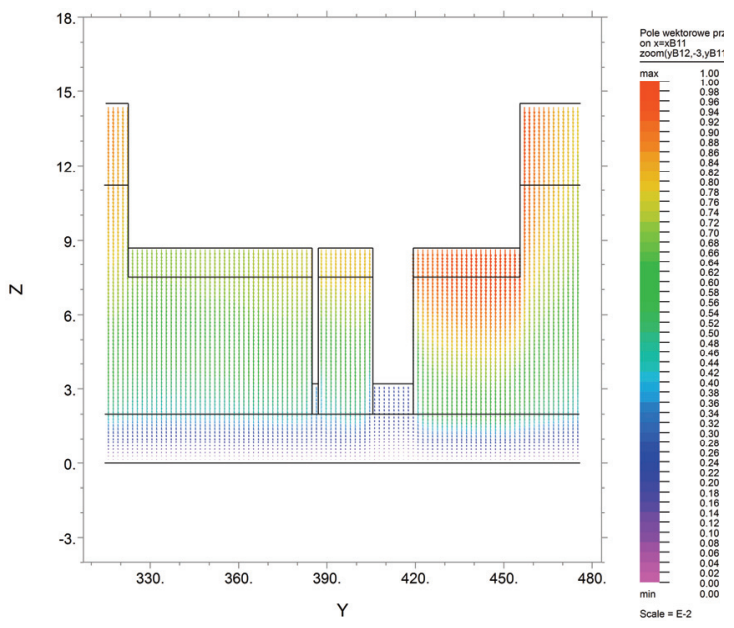

Fig. 13. Vectorial field of vertical displacements in the cross-section B1-B1'.

$($ Displacements are expressed in meters; scale multiplier $=1 \mathrm{e}-3)$

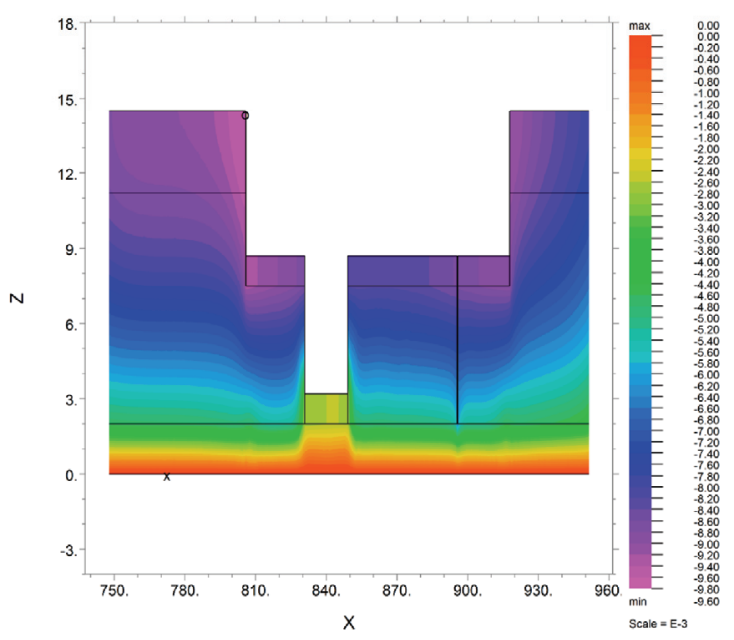

Fig. 14. The settlements of the system soil-slab in the cross-section A1-A1'.

(Settlements are expressed in meters; scale multiplier $=1 \mathrm{e}-3$ )
Separately, in Fig. 14 and Fig. 15, the settlements of the system soil-slab in the cross-sections A1-A1' and B1-B1' are presented, respectively.

These two last graphs show the significant variability of settlements which was already noted in the description of Fig. 9 and Fig. 10. Finally, we can follow the settlements of the system soil-slab as a function of time. To this purpose, the three points (two in the middle and one on the left) of the top surface of the slab were chosen. The settlements at these points, as a time function, are presented in Fig. 16.

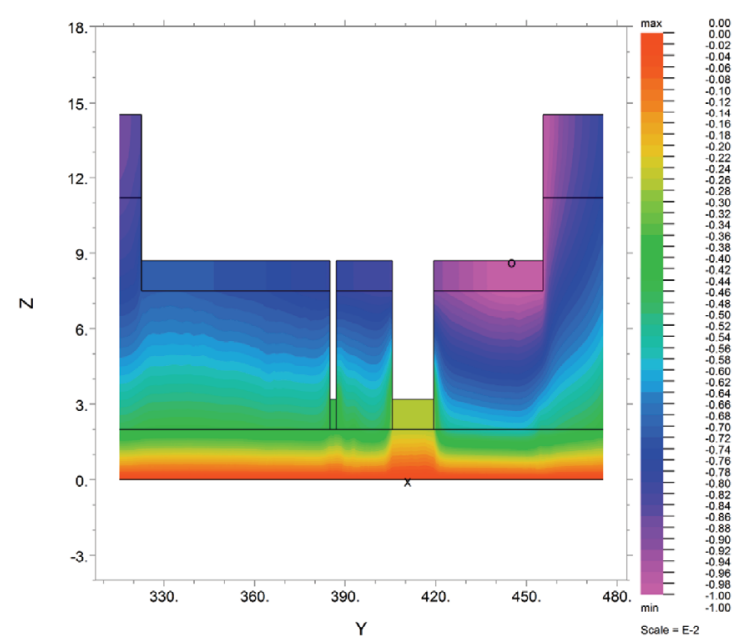

Fig. 15. The settlements of the system soil-slab in the cross-section B1-B1'.

(Settlements are expressed in meters; scale multiplier $=1 \mathrm{e}-2)$

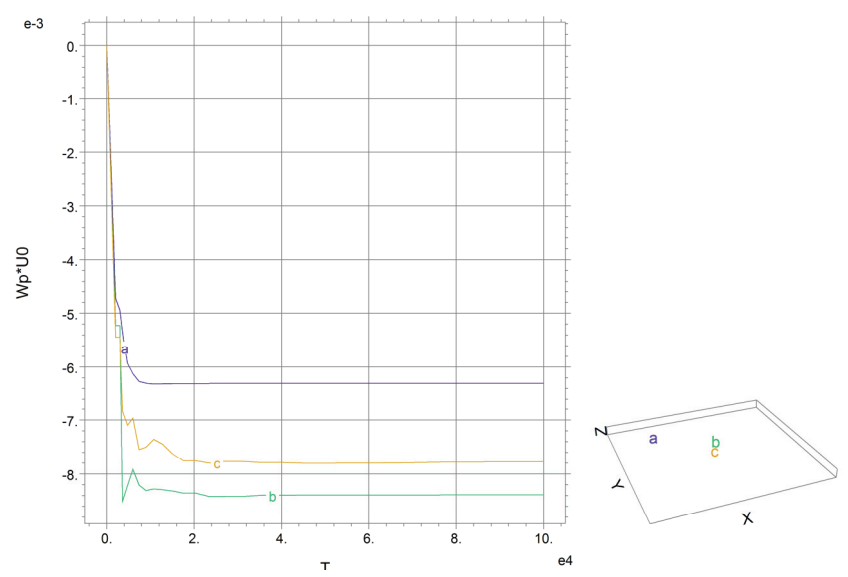

Fig. 16. The settlement of the top surface of the slab at three chosen points in time.

(Settlements are expressed in meters; scale multiplier $=1 \mathrm{e}-3$ )

\section{SUMMARY AND CONCLUSIONS}

In this work, the theoretical background of the consolidation model of Biot-Darcy has been presented. In its general form, this model takes into ac- 
count the movement of the liquid phase and the movement of solid phase (solid matrix) of a settling medium. It allows the determination of the stresses and strains, as well as settlements, for a threedimensional case.

In this paper, the authors have presented the application of this model in engineering practice. The case of a slab on a layered soil foundation, composed of sand with gravel and clay, was analyzed. The evolution of stresses $\sigma_{x x}, \sigma_{y y}, \sigma_{z z}$ in the slab and in the surrounding ground was determined under the assumption that the settlement process is quasi-static.

Next, an analysis of plastic potential using the Drucker-Prager criterion was conducted. The results indicate that there is no sign change of the potential yield. Therefore, there will be no plasticized zones. These results also confirm that the adoption of the mathematical model based on a linear stress-strain in this case is acceptable.

The distribution of horizontal displacements and settlement of the ground-slab system are illustrated in the corresponding cross-sections. In the latter case, the spatial image of the settlements of the baseplate is also presented. The diagrams show significant settlement differences at different points of the slab. This information may be very important if underground utilities are placed under the slab. Hence, the distribution of pipes of "lifeline" systems under the foundation seems a risky solution, which may threaten their sustainability. In contrast, the horizontal displacements of the slab are very small (to the order of $0.626 \mathrm{~mm}$ ).

The Biot-Darcy model also enables one to present the system displacement as a function of time, as illustrated by the corresponding example. The presented solution indicates that the use of a threedimensional model of consolidation in complex engineering problems is possible and provides additional benefits.

\section{REFERENCES}

[1] BartlewsKa M., Strzelecki T., Equations of Biots consolidation with Kelvin-Voight rheological frame, Studia Geotechnica et Mechanica, 2009, Vol. XXXI, No. 2.
[2] Baruce H., Madaune-Tort M., Saint-Macary P., On nonlinear Biot's consolidation models, Nonlinear Analysis, 2005, 63, e985-e995.

[3] Biot M.A., General Theory of three-dimensional Consolidation, J. Appl. Physics, 1941, Vol. 12.

[4] Biot M.A., Theory of Elasticity and Consolidation for a Porous Anisotropic Solid, Journal of Applied Physics, 1955, Vol. 26, No. 2, 182-185.

[5] Biot M.A., Theory of Propagation of Elastic Waves in a Fluid-Saturated porous Solid. I Low-Frequency Range, J.A.S.A., 1956, 28, 2, 168-178.

[6] Biot M.A., Willis D.G., The Elastic Coefficients of the Theory of Consolidations, Journal of Applied Physics, 1957, Vol. 24.

[7] Coussy O., Mechanics of Porous Continua, John Wiley \& Sons, 1995.

[8] FATT I., Compressibility of sandstones at low to moderate pressures, Bulletin of the American Association of Petroleum Geologists, 1924-1957, Vol. 42, No. 8, (August 1958).

[9] Ferronato M., Castelletto N., Gambolati G., A fully coupled 3-D mixed finite element model of Biot consolidation, Journal of Computational Physics, 2010, 229, 48134830.

[10] FlexPDE 6, Manual, www.pdesolutions.com

[11] Gaspar F.J., Lisbona F.J., VabishcheVich P.N., A finite difference analysis of Biot's consolidation model, Applied Numerical Mathematics, 2003, 44, 487-506.

[12] Menéndeza C., Nieto P.J.G., Ortega F.A., Bello A., Mathematical modelling and study of the consolidation of an elastic saturated soil with an incompressible fluid by FEM, Mathematical and Computer Modelling, 2009, 49, 2002 2018.

[13] Strzelecki T., Kostecki S., ŻAK. S., Modeling of flows through porous media, Lower Silesia Educational Publishers, 2008, (in Polish).

[14] Swan C., Lakes R., Brand R., Stewart K., Micromechanically based poroelastic modeling of fluid flow in Haversian bone, J. Biomech. Eng. 2003, 125, 25-37.

[15] Teatini P., Ferronato M., Gambolati G., Gonella M., Groundwater pumping and land subsidence in the EmiliaRomagna coastland, Italy: modeling the past occurrence and the future trend, Water Resour. Res., 2006, 42, DOI: 10.1029/2005WR004242.

[16] Herbert F. WANG, Theory of Linear Poroelasticity with Applications to Geomechanics and Hydrogeology, Princeton University Press, 2000.

[17] Folder with photos; https://www.google.pl/search?q=budowa + afrykarium + wroc $\% \mathrm{C} 5 \% 82 \mathrm{aw} \& \mathrm{tbm}=\mathrm{isch} \& \mathrm{tbo}=\mathrm{u} \&$ source $=$ un iv\&sa $=$ X\&ei $=$ H2JVKS_IojA7AbI6YDgCA\&ved $=0$ CC4QsA Q\&biw $=1536 \&$ bih $=709 \#$ facrc $=$ \&imgdii $=$ 